\title{
Resting-state functional changes in the precuneus within first-episode drug-naive patients with MDD
}

This article was published in the following Dove Press journal:

Neuropsychiatric Disease and Treatment

\author{
Gaizhi Li' \\ Kathryn Rossbach ${ }^{2}$ \\ Aixia Zhang' \\ Penghong Liu' \\ Kerang Zhang' \\ 'Shanxi Medical University, \\ Department of Psychiatry, First \\ Hospital of Shanxi Medical University, \\ Taiyuan, Shanxi, China; ${ }^{2}$ Independent \\ researcher, Atlanta, GA, USA
}

\begin{abstract}
Background: Major depressive disorder (MDD) is a leading psychiatric disorder that has a lack of biomarkers for a diagnosis.

Purpose: The objective of this study was to examine the structural and functional change in the precuneus within first-episode drug naive patients with MDD.

Methods: Thirty-two first episode drug-naive patients with MDD and thirty healthy controls (HCs) were recruited in this study; the structural MRI and fMRI data were collected using the 3.0 T Trio Siemens System. All the patients were interviewed using the HAMD-17.

Results: The difference between gray matter volume within the two groups was not observed. Results indicated that the low-frequency fluctuations (ALFF), fractional ALFF (fALFF) and regional homogeneity values of the precuneus within first-episode drug-naive patients with MDD were lower than the HCs. In addition, the fALFF value of the MDD was negatively and statistically significantly correlated with the HAMD-17 total score $(P<0.05)$.

Conclusion: The current study found abnormal activity of the precuneus at resting state in first-episode drug-naive patients with MDD, indicating that activity within the precuneus may be a potential biomarker for the diagnosis of MDD.
\end{abstract}

Keywords: major depressive disorder, resting-state fMRI, precuneus

\section{Introduction}

Major depressive disorder (MDD) is a heterogeneous disorder manifested by depressed mood, lack of energy, and loss of interest. The lifetime prevalence of MDD is approximately $15 \%-20 \%$, and the diagnosis of MDD is based on clinical symptoms, with a current lack of biological markers. Neuroimaging techniques are a noninvasive method that can be used to examine the brain structure and function. Numerous structural magnetic resonance imaging (SMRI) studies have sought to identify the key brain areas involved in the pathogenesis of MDD. ${ }^{1}$ Previous neuroimaging studies reported abnormal structure or function of certain brain areas, including the frontal lobe, precuneus, amygdala, and hippocampus; however, inconsistencies remain among the different studies due to small sample size, heterogeneous samples, different scanning parameters, different episodes, and various medications interacting with the studies.

Voxel-based morphometry (VBM) is a fully automated method for analyzing neuromorphological MRI data, which allows an unbiased investigation into the differences in brain structure between patients and healthy subjects. VBM has been widely used in characterizing subtle changes in brain structure in a variety of neurological and psychiatric diseases. ${ }^{2,3}$ A recent meta-analysis that combined 101 morphometric studies suggested that individuals with unipolar depression are characterized by volume reductions in the frontal cortex, orbitofrontal cortex, hippocampus, right cingulate cortex, caudate, and putamen and display an excess of white matter lesion volumes
Correspondence: Kerang Zhang Shanxi Medical University, Department of Psychiatry, First Hospital of Shanxi Medical University, No 85 Jiefang Road,

Taiyuan, Shanxi, China

Tel +86 I8I $35078 \mid 83$

Email krzhang_sxmu@vip.163.com 
in comparison with healthy controls (HCs). ${ }^{4}$ Another pooled meta-analysis and subgroup meta-analyses showed robustly reduced gray matter (GM) in the prefrontal and limbic regions in MDD, an increased right thalamus volume only found in first-episode medication-naive patients, and increased GM in the bilateral anterior cingulate cortex only in medication washout patients. ${ }^{5}$ However, these reports are inconsistent in detail, likely due to small sample sizes, heterogeneous subjects, and methodological differences.

Resting-state functional MRI (rs-fMRI) is a method of functional brain imaging that can be used to evaluate regional interactions that occur when a subject is not performing an explicit task. Several indexes can be measured by using an rs-fMRI. ${ }^{6,7}$ Amplitude of low-frequency fluctuations (ALFF) was developed as a power spectrum of low-frequency $(0.01-0.08 \mathrm{~Hz})$ fluctuations in the blood oxygen level-dependent signal, and fractional ALFF (fALFF) was developed as an improved approach to detect intrinsic spontaneous brain activity with higher sensitivity and specificity by Zou et al. ${ }^{8}$ Regional homogeneity ( $\mathrm{ReHo}$ ) is a voxel-based measure of brain activity which evaluates the similarity or synchronization between the time series of a given voxel and its nearest neighbors. ${ }^{9,10}$ Functional connectivity (FC) is a measure used for understanding the relationship between the activities in certain brain areas. The rs-fMRI is widely used in mental disorders, including MDD. ${ }^{11}$ Past studies have focused on the region of interest such as amygdala, hippocampus, and precuneus. Abnormal restingstate ALFF/fALFF/ReHo values observed in MDD patients may represent abnormal spontaneous neuronal activities, which may help researchers study the neural mechanism of MDD. ${ }^{12}$

The previous studies that used sMRI or fMRI (task-related or resting-state) to examine the brain structure of function changes of MDD for rs-fMRI generally used one of these parameters. In the current study, we aimed at examining the brain structure and function of patients with MDD using combined sMRI and rs-fMRI and whole brain analysis. We conducted sMRI and rs-fMRI scans in first-episode medication-naive patients with MDD and HCs to explore the brain structural and functional abnormality of patients with MDD using whole brain analysis, as well as the correlation between differentiated brain areas and depressive symptom.

\section{Materials and methods}

\section{Participants}

Thirty-two first-episode, right-handed treatment-naive adults with MDD and 30 HCs participated in this study. Patients were recruited from outpatient clinics at the First Hospital of Shanxi Medical University, Taiyuan, China from January 2011 to January 2012.
Criteria for depressed subjects were as follows: those 1) aged 25-50 years; 2) satisfied the Diagnostic and Statistical Manual of Mental Disorders, 4th Edition (DSM-IV) diagnostic criteria for MDD; 3) with first episode of depression; 4) who were medication-naïve; 5) who had no comorbid mental disorders; 6) who had the 17-item Hamilton Depression Rating Scale (HAM-D-17) score $>7$; 7) who had the Young Mania Rating Scale (YMRS) score $<5$; and 8) who had undergone outpatient treatment. Exclusion criteria for depressed patients were as follows: 1) those meeting criteria for any current or past DSM-IV Axis I disorder such as schizophrenia, schizoaffective disorder, bipolar disorder, or an anxiety disorder as a primary diagnosis; 2) those who had taken any prescription of psychotropic medications in the past 4 weeks; 3 ) those who were acutely suicidal; 4) those who were homicidal or requiring inpatient treatment; 5) those meeting criteria for substance dependence within the past year except for caffeine or nicotine; 6) those who had undergone positive urinary toxicology screening at baseline; 7) those who had drank alcohol in the past week; 8) those with serious medical or neurological illness; 9) those who were pregnant or breastfeeding at the time of the study; and 10) those with metallic implants or other contraindications to MRI.

HCs were recruited from January 2011 to January 2012 through flyers posted in public areas in Taiyuan, China. Criteria for healthy subjects were as follows: 1 ) those aged 25-50 years; 2) those with no history of psychiatric illness or substance abuse/ dependence; 3) those with no family history of major psychiatric or neurological illness in first-degree relatives; 4 ) those not taking any prescription or psychotropic medications at the time of the study; 5) those who had not drank alcohol in the past week; and 6) those with no serious medical or neurological illness. Exclusive criteria for HCs included the following: 1) those who were pregnant or breastfeeding and 2) those with metallic implants or other MRI contraindications. None of the HCs had a history of any neuropsychiatric disorders or personality disorders in this study.

This study's protocol was approved by the Institutional Review Board of the First Hospital of Shanxi Medical University, Taiyuan, China, and informed consent was obtained from all the participants. After explaining the protocol of the current study to the participants, they provided their informed consent by signing their names on the informed consent form. This informed consent indicated that all the participants were aware of the study protocol, including all risks of the study and the participants' ability to withdraw at any time.

\section{Clinical questionnaires}

The HAM-D-17 was developed by Hamilton in 1960, and a score of $0-7$ is considered to be normal, while a score of $\geq 20$ indicates a moderate severity level of depression. ${ }^{13}$ 
The HAM-D-17 was administered to assess the severity of depressive symptoms in this study. The YMRS is an 11-item multiple-choice diagnostic questionnaire that was used by psychiatrists to measure the severity of manic episodes in children and young adults. In the present study, it was administered to assess severity levels of mania/hypomania. The $\kappa$ score of the HAM-D-17 was 0.91 , indicating good interrater agreement, ${ }^{14}$ and the Cronbach's $\alpha$ score was 0.714 , indicating solid internal reliability. The $\kappa$ score and Cronbach's $\alpha$ score of YMRS was 0.93 and $0.66-0.92$, respectively. ${ }^{15}$

\section{Imaging acquisition and processing}

sMRI data were acquired from all the participants with a 3.0-T Trio Siemens System at the Shanxi Provincial People's Hospital, Taiyuan, China. One hundred and sixty dual echoes were scanned: repetition time $(\mathrm{TR})=2,300 \mathrm{~ms}$; effective echo times $(\mathrm{TE})=2.95 \mathrm{~ms}$; thickness $/$ skip $=1.2 / 0.6 \mathrm{~mm}$; sagittal slices $=160$; field of view $(F O V)=225 \times 240 \mathrm{~mm}^{2}$; matrix $=240 \times 256 \mathrm{~mm}^{2}$; and flip angle $(\mathrm{FA})=90^{\circ}$.

For the rs-fMRI, subjects were asked to rest quietly with their eyes closed during data acquisition; gradientecho single-shot echo planar imaging was used to acquire rs-fMRI image volumes with the same 3.0-T Trio System. We acquired 212 three-dimensional image volumes, each comprising 16 noncontiguous axial sections parallel to the intercommissural plane, with the following parameters: $\mathrm{TR}=2,000 \mathrm{~ms}$; $\mathrm{TE}=30 \mathrm{~ms}$; section thickness $=3 \mathrm{~mm}$; sagittal slices $=32 ; \mathrm{FOV}=240 \times 240 \mathrm{~mm}^{2}$; matrix $=64 \times 64 \mathrm{~mm}^{2}$; and $\mathrm{FA}=90^{\circ}$.

VBM 8 (http://dbm.neuro.uni-jena.de/vbm8/) was used for data preprocessing for T1 data, including the following steps: estimate and write, display one slice for all images, check sample homogeneity using covariance, and smooth data using Statistical Parametric Mapping 8 (SPM8) (http:// www.fil.ion.ucl.ac.uk/spm/) using an 8-mm full width at half-maximum (FWHM) Gaussian kernel. rs-fMRI data were preprocessed using Data Processing \& Analysis for
(Resting-State) Brain Imaging, ${ }^{16}$ including the following steps: remove the first 10 time points, slice timing, realign, normalize, smooth (using a 6-mm FWMH Gaussian kernel) and nuisance covariates regression (using whole brain signal and Friston 24). Detrend and bandpass temporal filtering $(0.01-0.08 \mathrm{~Hz}$ ) was used to reduce both physiological and high-frequency noises. For the ReHo score, the smoothing step was done after the calculation of ReHo. For ALFF/fALFF, a filter was not utilized. Any head motion of $>2.5 \mathrm{~mm}$ or an angular rotation of $>2.5^{\circ}$ in any direction was excluded. GM volume, ALFF, fALFF, and ReHo scores were tested for all the participants.

\section{Statistical analysis}

Clinical data were analyzed using SPSS Version 17 (SPSS Inc., Chicago, IL, USA), using an independent two-sample $t$-test between the MDD and HC groups. sMRI data were analyzed using SPM8, and the comparison of rs-fMRI data were analyzed using REST 1.8. Gaussian random field correction was used in this study, and the $P$-value was set as $<0.05$, using two-tailed tests. PickAtlas was utilized to make mask and REST 1.8 to extract the ALFF/fALFF/ ReHo values. ${ }^{17,18}$ The correlation between the HAM-D-17 and resting-state indexes was analyzed using SPSS Version 17.0. Partial correlation was used when the number of years of education was used as a covariate.

\section{Results}

\section{Demographic data}

For the VBM analysis, one patient with MDD and two HCs were excluded due to excessive head motion. The mean age of the MDD group was 33.13 \pm 9.77 years, and it was $32.96 \pm 9.33$ years for the HC group. No significant differences were observed for age or gender between the MDD and $\mathrm{HC}$ groups $\left(\mathrm{t}=0.066, \mathrm{df}=57, P=0.948 ; \chi^{2}=0.208, \mathrm{df}=1\right.$, $P=0.648$ ); however, the total number of years of education for patients with MDD was statistically significantly lower than that of HCs $(\mathrm{t}=-2.455, \mathrm{df}=57, P=0.018$; Table 1$)$.

Table I Demographic data of MDD and HC groups (VBM)

\begin{tabular}{llllll}
\hline Clinical variables & MDD $(\mathbf{n}=\mathbf{3 I})$ & HC $(\mathbf{n}=\mathbf{2 8})$ & $\mathbf{t} / \chi^{2}$ & $\mathbf{P}$-value & $\mathbf{9 5 \%} \mathbf{C l}$ \\
\hline Age & $33.13 \pm 9.77$ & $32.96 \pm 9.33$ & 0.066 & 0.948 & -4.829 to 5.158 \\
Gender (male/female) & $17: 14$ & $17: 11$ & 0.208 & 0.648 & -4.666 to -0.422 \\
Years of education & $15.74 \pm 4.8 \mathrm{I}$ & $18.28 \pm 3.02$ & -2.455 & 0.018 & \\
Age of onset & $32.33 \pm 9.74$ & & & \\
IIIness duration & $11.75 \pm 17.91$ & & & \\
HAM-D & $20.10 \pm 3.39$ & & & \\
HAM-A & $15.77 \pm 5.00$ & & & & \\
\hline
\end{tabular}

Abbreviations: HAM-A, Hamilton Anxiety Rating Scale; HAM-D, Hamilton Depression Rating Scale; HC, healthy control; MDD, major depressive disorder; VBM, voxelbased morphometry. 
Table 2 Demographic data of MDD and HC groups (rs-fMRI)

\begin{tabular}{llllll}
\hline Clinical variables & MDD $(\mathbf{n}=\mathbf{2 7})$ & $\mathbf{H C}(\mathbf{n}=\mathbf{2 7})$ & $\mathbf{t} / \chi^{2}$ & $\mathbf{P}$-value & $\mathbf{9 5 \%} \mathbf{C l}$ \\
\hline Age & $33.81 \pm 10.37$ & $32.18 \pm 9.02$ & 0.616 & 0.541 & -3.679 to 6.939 \\
Gender (male/female) & $14: 13$ & $15: 12$ & 0.074 & 0.785 & -4.975 to 0.432 \\
Years of education & $15.78 \pm 4.99$ & $18.48 \pm 3.12$ & -2.389 & 0.021 & \\
Age of onset & $32.85 \pm 10.37$ & & & \\
Illness duration & $10.02 \pm 16.24$ & & & \\
HAM-D & $20.62 \pm 3.22$ & & & \\
HAM-A & $15.69 \pm 5.18$ & & & & \\
\hline
\end{tabular}

Abbreviations: HAM-A, Hamilton Anxiety Rating Scale; HAM-D, Hamilton Depression Rating Scale; HC, healthy control; MDD, major depressive disorder; rs-fMRI, restingstate functional magnetic resonance imaging.

The comparison of VBM data was negative between the MDD and HC groups, regardless of whether educational years was utilized as a covariate or not.

Five patients with MDD and three HCs were excluded due to excessive head motion when the rs-fMRI data were analyzed. In terms of the rs-fMRI data, the mean age of the MDD group was 33.81 \pm 10.37 years, and it was 32.18 \pm 9.02 years for the HC group. No significant difference was observed for either age or gender between the MDD and HC groups $\left(\mathrm{t}=0.616, \mathrm{df}=52, P=0.541 ; \chi^{2}=0.074, \mathrm{df}=1\right.$, $P=0.785$ ), but the number of years of education for patients with MDD was lower than that of HCs $(\mathrm{t}=-2.389, \mathrm{df}=52$, $P=0.021$; Table 2).

Significant differences were observed between the MDD and HC groups for the rs-fMRI data, when it came to the following indexes: ALFF, fALFF, and ReHo. These values all included the precuneus (Brodmann area 23), and the values of the MDD group were found to be lower than those of the HC group. Further, to exclude the confounding factor, we reanalyzed the group difference with the number of years of education as a covariate; however, the differentiated brain areas were the same (Table 3; Figures 1-3).

Further, we extracted the ALFF/fALFF/ReHo scores from the precuneus and analyzed the correlations of the scores between the precuneus and the HAM-D-17 total score. The fALFF value of the precuneus was negatively and statistically significantly correlated with the HAMD-17 total score; however, the ALFF and ReHo values of the precuneus were not correlated with the HAM-D-17 total score (Table 4).

\section{Discussion}

In this study, we used sMRI and rs-fMRI to examine the GM volume and rs-fMRI indexes of first-episode drug-naive

Table 3 Differentiated brain areas between the MDD and HC groups (rs-fMRI)

\begin{tabular}{|c|c|c|c|c|c|c|c|c|}
\hline Index & Voxels & L/R & Brain areas & BA & $\mathbf{x}$ & y & $\mathbf{z}$ & $\begin{array}{l}\text { Peak } \\
\text { intensity }\end{array}$ \\
\hline \multirow[t]{7}{*}{ ALFF } & 155 & $\mathrm{R}$ & Precuneus & 23 & 9 & -42 & 39 & -3.8675 \\
\hline & 96 & L & Precuneus & & & & & \\
\hline & 65 & $\mathrm{R}$ & Middle cingulate cortex & & & & & \\
\hline & 53 & $\mathrm{R}$ & Calcarine & & & & & \\
\hline & 42 & L & Calcarine & & & & & \\
\hline & 42 & $\mathrm{R}$ & Lingual gyrus & & & & & \\
\hline & 34 & L & Cuneus & & & & & \\
\hline \multirow[t]{4}{*}{ fALFF } & 74 & $\mathrm{R}$ & Precuneus & 23 & 9 & -45 & 36 & -4.3126 \\
\hline & 41 & L & Precuneus & & & & & \\
\hline & 32 & $\mathrm{R}$ & Middle cingulate cortex & & & & & \\
\hline & 16 & L & Middle cingulate cortex & & & & & \\
\hline \multirow[t]{6}{*}{ ReHo } & 180 & $\mathrm{R}$ & Precuneus & 23 & 9 & -51 & 24 & -4.044 \\
\hline & 85 & L & Precuneus & & & & & \\
\hline & 41 & L & Posterior cingulate cortex & & & & & \\
\hline & 38 & $\mathrm{R}$ & Posterior cingulate cortex & & & & & \\
\hline & 29 & $\mathrm{R}$ & Middle cingulate cortex & & & & & \\
\hline & 23 & L & Middle cingulate cortex & & & & & \\
\hline
\end{tabular}

Abbreviations: ALFF, amplitude of low-frequency fluctuations; BA, Brodmann area; fALFF, functional ALFF; HC, healthy control; L, left; MDD, major depressive disorder; $\mathrm{R}$, right; ReHo, regional homogeneity; rs-fMRI, resting-state functional magnetic resonance imaging. 


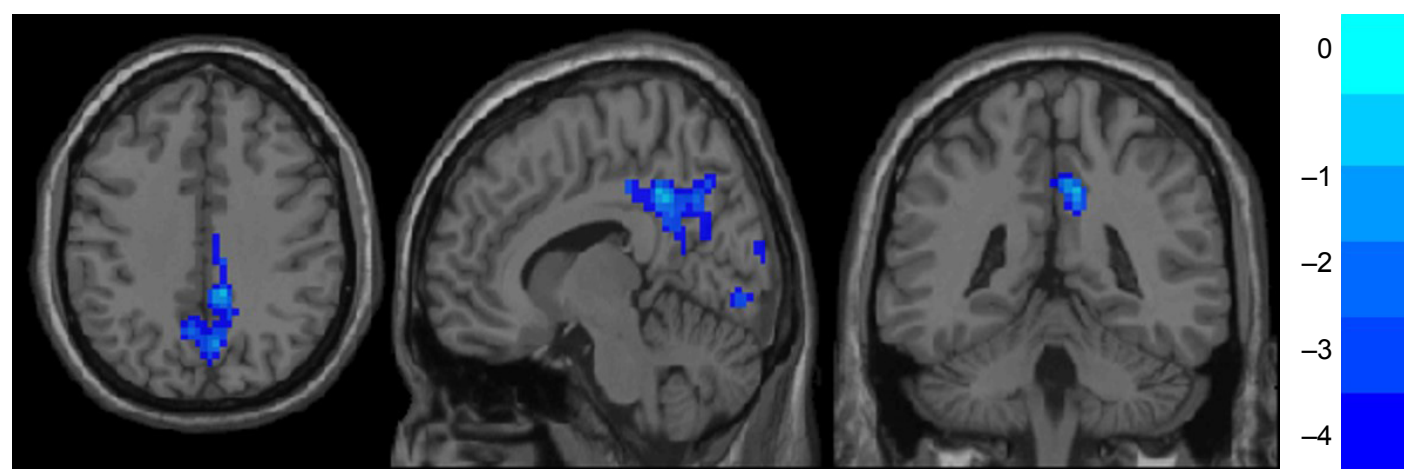

Figure I Lower ALFF value in the MDD group: bilateral precuneus, posterior cingulate cortex, right middle cingulate cortex, right calcarine, left calcarine, right lingual gyrus, and left cuneus.

Abbreviations: ALFF, amplitude of low-frequency fluctuations; MDD, major depressive disorder.

patients with MDD and HCs. The difference of the GM volume between the MDD and $\mathrm{HC}$ groups was not significant; however, the rs-fMRI indexes were found to be different between the MDD and HC groups. For the differentiated brain areas, the activity of the bilateral precuneus, middle cingulate cortex, calcarine, and right lingual gyrus was found to be lower in the MDD group than that in the HC group. In addition, the fALFF value of the precuneus was negatively correlated with the HAM-D-17 total score. Our findings suggested that when it came to the functional abnormalities of precuneus in the first-episode drug-native patients with MDD, the functional abnormalities could be potential biomarkers of MDD. While abnormal activity of the precuneus has been reported in MDD previously, the current study is the first study to document such abnormalities in an unconstrained whole brain study in medication-naïve, first-episode patients with MDD.

The structural differences were not observed between the MDD patients and HCs using whole brain analysis in this study. This finding is different from that of other reports. ${ }^{19}$ While previous studies reported the differentiated brain areas between MDD patients and $\mathrm{HCs}$, inconsistencies exist. Our study used whole brain analysis. The age and gender differences between MDD and HC groups were not significant, but the number of years of education within the MDD group was found to be statistically significantly lower than that in $\mathrm{HC}$ group. Finally, no significant difference was observed when the number of years of education was used as a covariate.

The precuneus is a central node of default mode network $(\mathrm{DMN}) .^{20}$ The posterior cingulate cortex $(\mathrm{PCC})$ is also a part of the DMN. Converging evidence suggests that a crucial role for the precuneus is the integration of mental processing through its role in cognitive control processes such as visual imagery, episodic memory, and self-directed operations. The precuneus has consistently been reported to be dysregulated as a neural substrate of depression. Our results showed that patients with MDD displayed lower activity in the precuneus, which suggests a possible neurobasis for MDD; this is consistent with the findings from two previous functional studies of depressed individuals. Jing et al also reported a decreased mean ALFF in the right precuneus and left lingual gyrus relative to the $\mathrm{HC}$ group. ${ }^{21}$

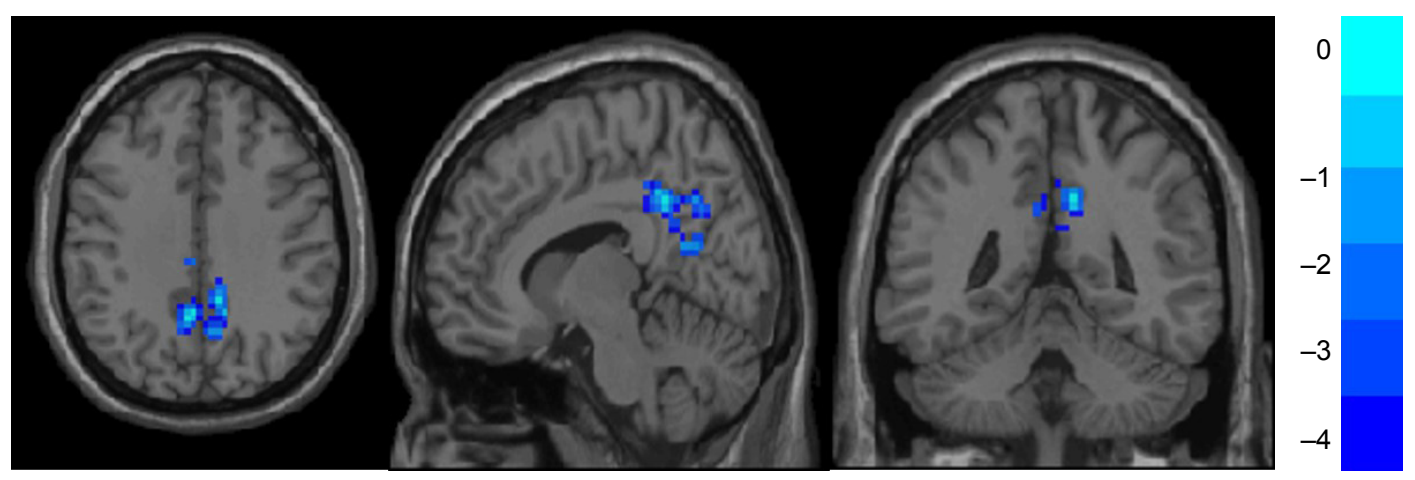

Figure 2 Lower fALFF value in the MDD group: bilateral precuneus and middle cingulate cortex. Abbreviations: fALFF, functional amplitude of low-frequency fluctuations; MDD, major depressive disorder. 


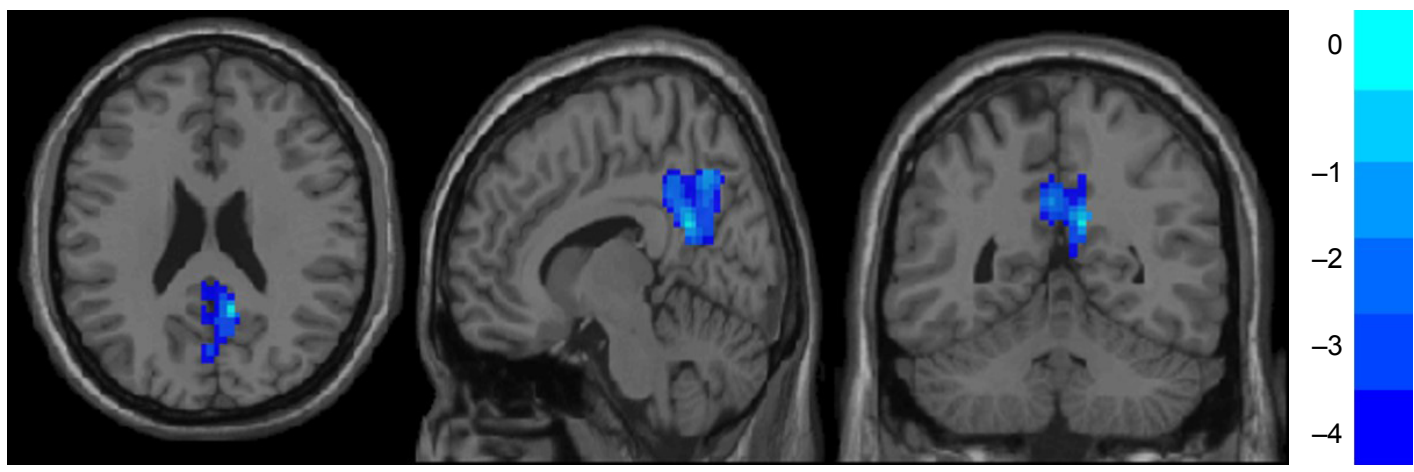

Figure 3 Lower ReHo value in the MDD group: bilateral precuneus, posterior cingulate cortex, and middle cingulate cortex. Abbreviations: MDD, major depressive disorder; ReHo, regional homogeneity.

The ALFF/fALFF/ReHo score of the middle cingulate cortex and PCC of MDD was found to be lower than those of the HCs. PCC/precuneus is a hub node for both structural and functional networks. ${ }^{22}$

In terms of other differentiated brain areas, the middle cingulate cortex is reported to be involved with emotion formation and processing, learning, and memory. The lower ALFF, fALFF, and ReHo values of the MCC may indicate an impairment within the patients with MDD; however, the correlation of the rs-fMRI index and the HAM-D-17 total score was not significant. Further, decreased functional connectivity strength has been observed in patients with MDD. ${ }^{23}$

Calcarine is the location of the visual primary cortex and related to visual information. Decreased short-range functional connectivity density was also reported in MDD by Zou et al. ${ }^{24}$ In addition, an increase or a decrease in ReHo was observed in the MDD patients. ${ }^{25}$

The lingual gyrus is linked to processing vision, especially related to letters. It is thought to also play a role in the analysis of logical conditions (ie, logical order of events) and encoding visual memories. Lower ALFF of the lingual gyrus was observed in this study, which is similar to a previous report by Wang et al. ${ }^{26}$

In our study, the FC was not examined, but the local activities of precuneus were examined using ALFF, fALFF, and ReHo scores. The fALFF score of the precuneus was

Table 4 Partial correlation of the HAM-D-I7 and indexes of rs-fMRI

\begin{tabular}{lllll}
\hline HAMD-17 & Covariates & ALFF & fALFF & ReHo \\
\hline HAM-D-17 & - & $-0.341 / 0.089$ & $-0.475 / 0.014^{*}$ & $-0.145 / 0.478$ \\
HAM-D-17 & $\begin{array}{l}\text { Years of } \\
\text { education }\end{array}$ & $-0.400 / 0.048^{*}$ & $-0.497 / 0.012^{*}$ & $-0.311 / 0.130$ \\
& & & \\
\hline
\end{tabular}

Note: $* P<0.05$

Abbreviations: ALFF, amplitude of low-frequency fluctuations; fALFF, functional ALFF; HAM-D-17, 17-item Hamilton Depression Rating Scale; ReHo, regional homogeneity; rs-fMRI, resting-state functional magnetic resonance imaging. negatively correlated with the HAM-D-17; however, the value of the ALFF/ReHo was not correlated with the severity of depression, indicating that the lower fALFF value is correlated with more severe depressive symptoms. In a meta-analysis conducted by Liu et al, patients with recurrent MDD exhibited decreased fALFF in the right posterior insula and right precuneus, increased fALFF in the left ventral anterior cingulate cortex, and decreased fALFF in the right precuneus, and increased fALFF in the right middle insula was correlated with the number of depressive episodes in the recurrent MDD groups and remitted MDD groups. ${ }^{28}$ Our results are fairly similar to the results of this meta-analysis, as we found lower fALFF value in the right precuneus, as well as a correlation between the value of decreased fALFF and the number of depressive episodes. As our study only included first-episode patients with MDD, the correlation of fALFF and episodes was not examined, but the decreased fALFF was correlated with more severe depressive symptoms. Peng et al investigated the $\mathrm{FC}$ of the precuneus and found that a greater severity of depression was associated with greater anticorrelation between the precuneus and the temporoparietal junction as well as stronger positive connectivity between the precuneus and the dorsomedial prefrontal cortex. ${ }^{27}$ Though different indexes were used in these studies, the abnormal function of precuneus is involved in the pathophysiology of MDD.

\section{Limitations}

This study examined the resting-state activity of patients with MDD and reported a possible biological marker within MDD; however, there are several limitations. First, this is a cross-sectional study, and future studies are needed to confirm the longitudinal changes of resting-state brain activity. Second, the current study utilized a relatively small sample size; thus, a larger sample size would generate more generalizable results. Lastly, the number of years of education 
within the MDD group was found to be slightly lower than that in the $\mathrm{HC}$ group, which could pose a problem when analyzing differences between the two groups. Nonetheless, our results remained consistent when we reanalyzed our data using the number of years of education as a covariate. Further, other researchers reported that females with high body mass indexes (BMIs) had increased low-frequency activity in clusters located in the putamen, claustrum, and insula compared with healthyweight controls. We did not analyze the correlation of BMI and rs-fMRI index in the current study. ${ }^{29}$ In addition, the current study focused on patients with MDD, whereas other studies have reported the difference between MDD and bipolar depression; we plan on conducting future studies to focus on this difference. ${ }^{30}$

\section{Conclusion}

While the GM volume of first-episode, drug-naive patients with MDD may be the same as that of HCs within the current study, the resting-state activity of bilateral precuneus may be an important biological marker of patients with MDD. These findings shed light on the pathogenesis underlying MDD and imply that using an rs-fMRI with ALFF/fALFF/ReHo analysis as a research and clinical tool may be a suggestive and useful marker in monitoring cerebral dysfunction in MDD.

\section{Acknowledgments}

We thank all the volunteers who participated in this study. This study was funded by the National Natural Science Foundation of China (No 81471379). This study is also funded by the Postdoctoral Foundation of Shanxi Medical University, Doctoral Foundation of Shanxi Medical University and Doctoral Foundation of Shanxi Province.

\section{Author contributions}

GL and KZ designed the study. GL analyzed the MRI data and wrote this manuscript. KZ was funded by the foundation. $\mathrm{AZ}$ and PL collected the MRI and clinical data. KR revised the manuscript. All authors contributed toward data analysis, drafting and revising the paper and agree to be accountable for all aspects of the work.

\section{Disclosure}

The authors report no conflicts of interest in this work.

\section{References}

1. Graham J, Salimi-Khorshidi G, Hagan C, et al. Meta-analytic evidence for neuroimaging models of depression: state or trait? J Affect Disord. 2013;151(2):423-431.

2. Palaniyappan L, Maayan N, Bergman H, Davenport C, Adams CE, Soares-Weiser K. Voxel-based morphometry for separation of schizophrenia from other types of psychosis in first episode psychosis. Cochrane Database Syst Rev. 2015;(8):CD011021.
3. Rogers JC, De Brito SA. Cortical and subcortical gray matter volume in youths with conduct problems: a meta-analysis. JAMA Psychiatry. 2016;73(1):64-72.

4. Lee MH, Smyser CD, Shimony JS. Resting-state fMRI: a review of methods and clinical applications. AJNR Am J Neuroradiol. 2013; 34(10):1866-1872.

5. Guerra-Carrillo B, Mackey AP, Bunge SA. Resting-state fMRI: a window into human brain plasticity. Neuroscientist. 2014;20(5):522-533.

6. Arnone D, Mcintosh AM, Ebmeier KP, Munafò MR, Anderson IM Magnetic resonance imaging studies in unipolar depression: systematic review and meta-regression analyses. Eur Neuropsychopharmacol. 2012;22(1):1-16.

7. Zhao YJ, Du MY, Huang XQ, et al. Brain grey matter abnormalities in medication-free patients with major depressive disorder: a metaanalysis. Psychol Med. 2014;44(14):2927-2937.

8. Zou QH, Zhu CZ, Yang Y, et al. An improved approach to detection of amplitude of low-frequency fluctuation (ALFF) for resting-state fMRI: fractional ALFF. J Neurosci Methods. 2008;172(1):137-141.

9. Zang Y, Jiang T, Lu Y, He Y, Tian L. Regional homogeneity approach to fMRI data analysis. Neuroimage. 2004;22(1):394-400.

10. Kendall MG, Gibbons JD. Rank Correlation Methods. London: Edward Arnold View; 1990.

11. Zhou M, Hu X, Lu L, et al. Intrinsic cerebral activity at resting state in adults with major depressive disorder: a meta-analysis. Prog Neuropsychopharmacol Biol Psychiatry. 2017;75:157-164.

12. Li W, Chen Z, Wu M, et al. Characterization of brain blood flow and the amplitude of low-frequency fluctuations in major depressive disorder: a multimodal meta-analysis. $J$ Affect Disord. 2017;210:303-311.

13. Hamilton M. A rating scale for depression. J Neurol Neurosurg Psychiatry. 1960;23:56-62.

14. Jinping Z, Yanping Z. The reliability and validity of Hamilton Depression Rating Scale. Chin Ment Health J. 1992;6(5):214-216.

15. Young RC, Biggs JT, Ziegler VE, Meyer DA. A rating scale for mania: reliability, validity and sensitivity. B J Psychiatry. 1978;133: 429-435.

16. Yan CG, Wang XD, Zuo XN, Zang YF. DPABI: Data Processing \& Analysis for (Resting-State) Brain Imaging. Neuroinformatics. 2016; 14(3):339-351.

17. Song XW, Dong ZY, Long XY, et al. REST: a toolkit for resting-state functional magnetic resonance imaging data processing. PLoS One. 2011;6(9):e25031.

18. Maldjian JA, Laurienti PJ, Burdette JH. Precentral gyrus discrepancy in electronic versions of the Talairach atlas. Neuroimage. 2004;21(1): 450-455.

19. Bora E, Fornito A, Pantelis C, Yücel M. Gray matter abnormalities in major depressive disorder: a meta-analysis of voxel based morphometry studies. J Affect Disord. 2012;138(1-2):9-18.

20. Raichle ME, MacLeod AM, Snyder AZ, Powers WJ, Gusnard DA, Shulman GL. A default mode of brain function. Proc Natl Acad Sci U S A. 2001;98(2):676-682.

21. Jing B, Liu CH, Ma X, et al. Difference in amplitude of low-frequency fluctuation between currently depressed and remitted females with major depressive disorder. Brain Res. 2013;1540:74-83.

22. van den Heuvel MP, Sporns O. Network hubs in the human brain. Trends Cogn Sci. 2013;17(12):683-696.

23. Wu H, Sun H, Xu J, et al. Changed hub and corresponding functional connectivity of subgenual anterior cingulate cortex in major depressive disorder. Front Neuroanat. 2016;10:120.

24. Zou K, Gao Q, Long Z, et al. Abnormal functional connectivity density in first-episode, drug-naive adult patients with major depressive disorder. J Affect Disord. 2016;194:153-158.

25. Shen Z, Jiang L, Yang S, et al. Identify changes of brain regional homogeneity in early and later adult onset patients with first-episode depression using resting-state fMRI. PLoS One. 2017;12(9):e0184712.

26. Wang L, Dai W, Su Y, et al. Amplitude of low-frequency oscillations in first-episode, treatment-naive patients with major depressive disorder: a resting-state functional MRI study. PLoS One. 2012;7(10):e48658. 
27. Peng D, Liddle EB, Iwabuchi SJ, et al. Dissociated large-scale functional connectivity networks of the precuneus in medication-naive first-episode depression. Psychiatry Res. 2015;232(3):250-256.

28. Liu CH, Ma X, Yuan Z, et al. Decreased resting-state activity in the precuneus is associated with depressive episodes in recurrent depression. J Clin Psychiatry. 2017;78(4):e372-e382.

29. Hogenkamp PS, Zhou W, Dahlberg LS, et al. Higher resting-state activity in reward-related brain circuits in obese versus normal-weight females independent of food intake. Int J Obes (Lond). 2016;40(11): $1687-1692$.
30. Serafini G, Pompili M, Borgwardt S, et al. Brain changes in earlyonset bipolar and unipolar depressive disorders: a systematic review in children and adolescents. Eur Child Adolesc Psychiatry. 2014;23(11): 1023-1041.

\section{Publish your work in this journal}

Neuropsychiatric Disease and Treatment is an international, peerreviewed journal of clinical therapeutics and pharmacology focusing on concise rapid reporting of clinical or pre-clinical studies on a range of neuropsychiatric and neurological disorders. This journal is indexed on PubMed Central, the 'PsycINFO' database and CAS, and is the official journal of The International Neuropsychiatric Association (INA). The manuscript management system is completely online and includes a very quick and fair peer-review system, which is all easy to use. Visit http://www.dovepress.com/testimonials.php to read real quotes from published authors.

Submit your manuscript here: http://www.dovepress.com/neuropsychiatric-disease-and-treatment-journal 